\title{
Beam manipulation for resonant plasma wakefield acceleration
}

\author{
E. Chiadroni ${ }^{\mathrm{a}, *}$, D. Alesini ${ }^{\mathrm{a}}$, M.P. Anania ${ }^{\mathrm{a}}$, A. Bacci ${ }^{\mathrm{b}}$, M. Bellaveglia ${ }^{\mathrm{a}}$, A. Biagioni ${ }^{\mathrm{a}}$, F.G. Bisesto ${ }^{\mathrm{a}}$, \\ F. Cardelli ${ }^{\mathrm{a}}$, G. Castorina ${ }^{\mathrm{a}}$, A. Cianchi ${ }^{\mathrm{c}}$, M. Croia ${ }^{\mathrm{a}}$, A. Gallo ${ }^{\mathrm{a}}$, D. Di Giovenale ${ }^{\mathrm{a}}$, G. Di Pirro ${ }^{\mathrm{a}}$, \\ M. Ferrario ${ }^{a}$, F. Filippi $^{\mathrm{d}}$, A. Giribono ${ }^{\mathrm{d}}$, A. Marocchino $^{\mathrm{a}}$, A. Mostacci $^{\mathrm{d}}$, M. Petrarca $^{\mathrm{d}}$, L. Piersanti $^{\mathrm{a}}$, \\ S. Pioli ${ }^{a}$, R. Pompili ${ }^{\mathrm{a}}$, S. Romeo ${ }^{\mathrm{a}}$, A.R. Rossi ${ }^{\mathrm{b}}$, J. Scifo ${ }^{\mathrm{a}}$, V. Shpakov ${ }^{\mathrm{a}}$, B. Spataro ${ }^{\mathrm{a}}$, A. Stella ${ }^{\mathrm{a}}$, \\ C. Vaccarezza ${ }^{a}, F$. Villa ${ }^{a}$ \\ a INFN-Laboratori Nazionali di Frascati, via E. Fermi 40, 00044 Frascati, Italy \\ b INFN-Milano, Via Celoria 16, 20133 Milano, Italy \\ c INFN-Roma Tor Vergata and Università di Roma Tor Vergata, Via della Ricerca Scientifica 1, 00133 Roma, Italy \\ d INFN-Roma 1 and Università di Roma La Sapienza, Piazzale A. Moro 2, 00185 Roma, Italy
}

\section{A R T I C L E I N F O}

Keywords:

PWFA

Laser comb technique

Transformer ratio

\begin{abstract}
A B S T R A C T
Plasma-based acceleration has already proved the ability to reach ultra-high accelerating gradients. However the step towards the realization of a plasma-based accelerator still requires some effort to guarantee high brightness beams, stability and reliability. A significant improvement in the efficiency of PWFA has been demonstrated so far accelerating a witness bunch in the wake of a higher charge driver bunch. The transformer ratio, therefore the energy transfer from the driver to the witness beam, can be increased by resonantly exciting the plasma with a properly pre-shaped drive electron beam. Theoretical and experimental studies of beam manipulation for resonant PWFA will be presented here.
\end{abstract}

\section{Introduction}

Plasma-based accelerators represent the new frontier for the development of compact advanced radiation sources and next generation linear colliders. Plasma-based acceleration has already proved the ability to reach ultra-high accelerating gradients. In the last decades great progresses have been achieved in several international laboratories $[1-5]$ to demonstrate the acceleration of electron beams with accelerating gradients of the order of several tens of $\mathrm{GV} / \mathrm{m}$, as produced by either laser-driven or particle-driven plasma wakefields, LWFA or PWFA, respectively.

The main issue, so far still not achieved, concerns the quality of the accelerated electron beam. The figure of merit of electron beams is the so-called brightness, which represents the capability of a beam, made of a large $\left(\approx 10^{9}\right)$ number of quasi-monochromatic electrons to be focused on a very small transverse area. In terms of beam parameters, it translates into $\mathrm{mm}$ mrad scale transverse normalized emittance and $0.1 \%$ energy spread. High brightness electron beams are the future goal of such kind of particle accelerators in order to compete with those based on conventional RF photo-injectors. The current goal of the worldwide plasma, laser and photo-injector communities is to demonstrate the stable and repeatable acceleration of high brightness beams.
The next step is then the extraction and transport of the beam, preserving its quality, i.e. 6D high brightness, stability and reliability to drive a plasma-based user facility [6-8].

In PWFA the high-gradient wakefield is driven by an intense, highenergy charged particle beam, named as driver beam, as it passes through the plasma. The space-charge forces of the electron bunch blow out the plasma electrons, which rush back in and overshoot setting up plasma oscillations, at a frequency $\omega_{p}=\sqrt{\frac{n_{0} e^{2}}{\varepsilon_{0} m_{e}}}$, depending on the unperturbed plasma density, $n_{0} ; e$ and $m_{e}$ are the electron elementary charge and mass, respectively. The plasma response to the perturbation introduced by the electron beam can be expressed by the degree of nonlinearity of the plasma dynamics that defines the plasma wave regime. The figures of merit are the beam charge in the cubic plasma skin-depth, $k_{p}^{-3}$, the so-called reduced charge, $\widetilde{Q}=\frac{N_{b} k_{p}^{3}}{n_{0}}$, and $\alpha=\frac{n_{b}}{n_{0}}$, where $N_{b}$ is the driver number of electrons and $n_{b}$ the beam density $[9,10]$. The quasi non-linear regime [11] is defined by a linear response of the plasma, $\widetilde{Q}<1$, combined to the bubble formation in an under-dense plasma $(\alpha>1)$. In the following we will consider the quasi non-linear or weakly non-linear, $\widetilde{Q} \approx 1$, regime.

When a single bunch interacts with the plasma, the head of the bunch transfers a large fraction of its kinetic energy to the plasma. The

\footnotetext{
* Corresponding author.

E-mail address: enrica.chiadroni@lnf.infn.it (E. Chiadroni).
} 
plasma, acting as an energy transformer, transfers the gained energy to the tail of the bunch, resulting in an ultimate beam energy spread of $100 \%$ [3]. In order to minimize the beam energy spread, which would contribute to spoil the overall brightness, a second, appropriately phased accelerating beam, named as witness beam, and containing fewer particles than the drive beam, is then accelerated by the wake. This 2-bunch configuration allows to strongly decrease the energy spread of the accelerated witness bunch down to few \% [6]. Acting as an energy transformer, PWFA has the great potential to double the beam energy in a single stage [3]. The energy transfer from the drive bunch to the plasma is optimized by maximizing the transformer ratio $R=\left|\frac{E_{+, \max }}{E_{-}}\right|$, defined as the ratio between the maximum accelerating field behind the drive bunch, $E_{+, \max }$, where the particles of the witness bunch can be placed, and the maximum decelerating field within the drive bunch, $E_{-, \max } . R$ quantifies the energy gain of a witness bunch placed at the accelerating phase. However the Wakefield Fundamental Theorem [12,13] sets a limit to the maximum energy gain in PWFA. Indeed, for a symmetric drive bunch, the transformer ratio reaches the maximum value of 2 , when the maximum retarding wake occurs at the center of the bunch. The drive beam energy is depleted after a distance $L_{d} \sim \gamma_{b} m_{e} c^{2} / e E_{-, \max }$, with $\gamma_{b}$ being the Lorentz factor for the driver beam, while the witness bunch gains $\Delta \gamma m_{e} c^{2} \sim e E_{+, \max } L_{d}=R \gamma_{b} m_{e} c^{2}$. In case of Gaussian bunches, if the longitudinal matching condition, $k_{p} \sigma_{z}=\pi / 2$, is satisfied, and the beam loading effect is negligible, then $R=2$ and the witness beam energy is doubled. This result is also obtained in case of a symmetric train of $N=4$ Gaussian bunches, as shown in Fig. 1: the maximum wake is obtained when the spacing equals the plasma wavelength, $\lambda_{p}$, and the final wake amplitude scales linearly with the amplitude of the first driver bunch, i.e. $E_{f}=N E_{1}$. Simulations are performed by means of a hybrid kinetic-fluid code, called Architect [14-16], in the weakly non-linear regime $(\widetilde{Q}=0.75$ and $\alpha>1$ ).

\section{Resonant PWFA for enhancing the transformer ratio}

By properly tailoring the driver bunch shape, the witness beam energy can be more than doubled when the charge distribution is shaped such that all particles in the bunch see the same retarding field, resulting in $R>2$ [17]. Therefore, asymmetric drive bunch current profiles, i.e. triangular, double triangle, doorstep-like distributions, overcome this limit $[18,19]$, because all longitudinal slices lose energy at the same rate. The same result is also obtained by means of multiple ramped bunch trains, which excite resonant plasma wakes [20].

By placing multiple drive bunches at the accelerating phase of the wakefield driven by preceding bunches, then by adjusting the charge in each bunch, it is possible either to optimize the accelerating gradient (Fig. 2) or the transformer ratio of PWFA (Fig. 3). The use of a train of bunches allows to gently transfer the energy first from one bunch to the

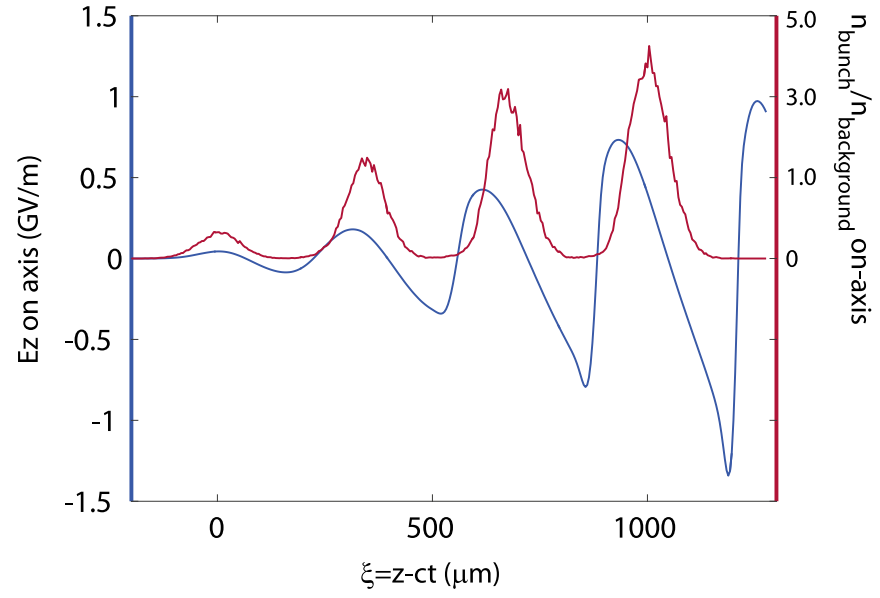

Fig. 2. Longitudinal electric field on axis (blue curve) and normalized bunch density profile (red curve), $\alpha=n_{b} / n_{0}$, for a ramped charge profile (ratio 1:3:5:7) in the weakly non-linear regime: $\alpha \gg 1$ and $\widetilde{Q}=0.75$. The driver bunch spacing is $\lambda_{p}$ and the calculated transformer ratio is $R=3$. (For interpretation of the references to color in this figure caption, the reader is referred to the web version of this paper.)

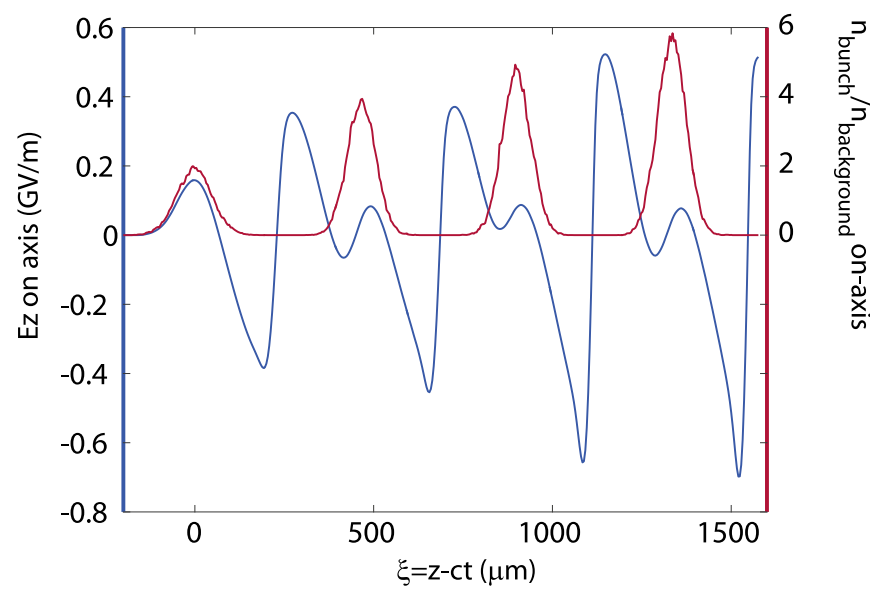

Fig. 3. Longitudinal electric field on axis (blue curve) and normalized bunch density profile (red curve), $\alpha=n_{b} / n_{0}$, for a ramped charge profile (ratio 1:1.6:2:2.4) in the nonlinear regime: $\alpha \gg 1$ and $\widetilde{Q}=1.4$. The driver bunch spacing is $1.5 \lambda_{p}$, in order to increase the transformer ratio: $R=8$. (For interpretation of the references to color in this figure caption, the reader is referred to the web version of this paper.)

following then to the resulting wake, piling up the total energy. A unique symmetric bunch would have part of the energy uniquely dispersed into the self-induced bubble. The multi-bunch distribution has an extra advantage: since the energy depletion for each bunch

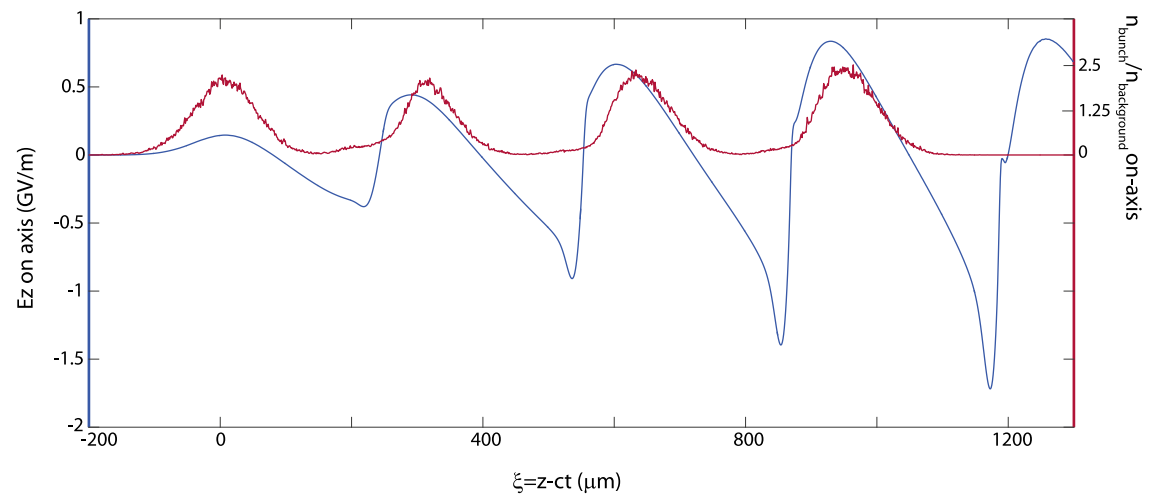

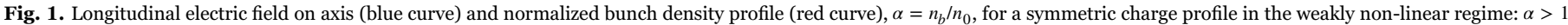

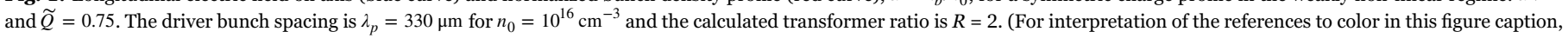
the reader is referred to the web version of this paper.) 
occurs at constant pace, they retain the phase separation over evolution. The advantage is to pile up energy with less energy dissipation. As shown in Fig. 2, four driver bunches with $\sigma_{z}<\lambda_{p} / 4$, whose charges scale as 1:3:5:7 drive a more than $1 \mathrm{GV} / \mathrm{m}$ wake when they are placed $\lambda_{p}=330 \mu \mathrm{m}$ apart $\left(n_{0}=10^{16} \mathrm{~cm}^{-3}\right)$. Placing the bunch at a $\lambda_{p}$ corresponds to where the bubble closes. It means that a negative charge is added to the bubble closure where the negative charge naturally accumulates, resulting in pumping the field without an efficient mechanism to decrease the decelerating field, necessary to increase the transformer ratio. A case of interest at SPARC_LAB in the weakly non-linear regime $(\widetilde{Q}=0.75, \alpha \gg 1)$ is shown in Fig. 2, where the resulting transformer ratio is $R=3$.

To increase the transformer ratio in the case of a train of electron bunches, they have to be placed at phase-opposition. As Fig. 3 shows, each bunch is placed at a longitudinal position where the accelerating electric field underneath the drivers is reduced, i.e. at $1.5 \lambda_{p}\left(\lambda_{p}=\right.$ $330 \mu \mathrm{m}$ at $10^{16} \mathrm{~cm}^{-3}$ plasma density). The estimated transformer ratio for this case of interest at SPARC_LAB is around 8. It means that the incoming energy is increased by 8 times in a single PWFA stage. The operation regime is between weakly non-linear and non-linear, defined by a reduced charge $\widetilde{Q}=1.4$ and $\alpha \gg 1$, for the total charge $350 \mathrm{pC}$, distributed over four driver bunches. Such a transformer ratio is possible by means of ramped bi-Gaussian profiles, with charge scaling as 1:1.6:2:2.4 and separation of $1.5 \lambda_{p}$. The wakefield under each bunch can be made equal, thus increasing the transformer ratio beyond two, which now scales linearly with the number of bunches, $N$.

\section{Multi-bunch shaping for resonant PWFA experiments}

Several methods can be used to longitudinally shape electron beams. Some of them have been theoretically studied, e.g. linearly ramped current profiles combined with a door-step or exponential initial distribution [21]; some others experimentally investigated, e.g. train of drive bunches (instead of a shaped single drive bunch) with appropriate charge and temporal separation [22-24].

A ramped bunch train can be shaped via photo-emission exploiting the so-called laser comb technique [25], which combines laser pulse shaping and beam dynamics manipulation based on velocity bunching compression. A multi-bunch train, resembling to a comb, therefore named as laser comb, can be produced directly at the photo-cathode, exploiting the fast response of the cathode material. A single laser pulse is split into two or more orthogonally polarized pulses by means of a $\alpha$ BBO birefringent crystal. Because of the polarization dependent path, the two polarizations are delayed in time of a quantity depending on the crystal thickness, i.e. $\Delta \tau=\left|\frac{1}{v_{g e}}-\frac{1}{v_{g o}}\right| L_{\text {crystal }}$. Therefore, by stacking several crystals, one after the other, it is possible to generate a train of $\mathrm{N}$-bunches. Delay lines can be used to fully control the bunch interdistance, while half-wave plates (HWP) unbalance the laser intensity to allow ramps of charge [26]. When a train of laser pulses illuminates the photo-cathode, because of the metal fast response, a train of electron bunches is produced. Because of longitudinal space charge forces, the longitudinally modulated beam acquires downstream the gun an energy modulation. Therefore, when injected in the first accelerating section, operating as RF compressor in the so-called Velocity Bunching (VB) regime $[27,28]$, the energy modulation can be transformed back into a density modulation. The regime suitable for the resonant PWFA application, i.e. where the sub-bunches are well separated and their distance can be tuned with the RF compressor injection phase, is called deep over-compression: the RF phase of the first accelerating structure is set to produce a longitudinal over-focusing, and the comb generated beam at the cathode recovers the initial density modulation. The final pulses inter-distance can be adjusted in the sub-picoseconds range by the $\mathrm{RF}$ compression phase and the temporal structure is usually frozen at the exit of the linac. Since during the VB process the longitudinal phase space (LPS) is rotated, the position of bunches is reversed at the end of the process. Therefore, since the witness follows the drivers in the plasma wake, the witness bunch is extracted from the cathode at a phase earlier than the drivers. The main advantage of the laser comb technique consists in the high versatility with no cut of charge and the possibility of tuning beam parameters, i.e. charge, duration and time spacing through laser shaping and RF compression phase.

A different approach to produce trains of ps-spaced bunches relies on collimation: a multi-wire mask is placed in a high dispersion, lowbeta function region of a dogleg beam line in order to produce a temporal bunch train out of a long bunch with a correlated energy spread [23]. A THz repetition rate bunch train can also be generated with a transversely segmented beam (with a multi-slit mask) via a transverse-to-longitudinal phase space exchange technique, obtained placing a deflecting cavity between two dispersive sections [29]. Both techniques have the disadvantage to lose charge, cut by the mask, and to allow fine adjustment of time spacing by only changing the mask geometry. However, the multi-slit mask has the great advantage to shape triangular driver bunches in the train.

\section{Beam manipulation for resonant PWFA experiments at SPARC_LAB}

One of the most promising candidates for high gradient PWFA acceleration and efficiency consists in a plasma channel driven by a resonant bunch train. At the SPARC_LAB test facility [30], this scheme will be investigated by injecting a low emittance witness beam, produced by the high brightness SPARC photo-injector [31], into a pre-formed plasma channel [32], excited by a train of short, fs-scale, driver bunches with constant and/or ramped charge distribution [22]. In this case the beam quality of the accelerated witness bunch depends on the injector performance and on the capability to mitigate beam degradation in the plasma with a proper matching of the beam with the plasma [33].

SPARC_LAB hosts a high brightness photo-injector to drive, together with a high power laser ( $200 \mathrm{TW},<30 \mathrm{fs}$ pulse), Thomson back-scattering [34] and THz radiation [35,36] sources, Free-Electron Laser (FEL) experiments [37-39] and plasma-based acceleration experiments, both laser-driven [40] and particle-driven [41]. An update layout is shown in Fig. 4: the third S-band traveling wave (TW) accelerating structure has been replaced with a TW constant impedance C-band structure, and the interaction chamber, fully equipped with diagnostics, both transverse and longitudinal, based on Electro-Optical sampling [42] and THz radiation [43,44], with a $\mathrm{H}_{2}$ plasma discharge capillary [32] and permanent quadrupole magnets for beam matching in and out from the plasma. The C-band structure, fed by a Toshiba C-band klystron, a high voltage pulsed modulator (from ScandiNova) and a $400 \mathrm{~W}$ solid state driver for the klystron, is $1.4 \mathrm{~m}$ long (including couplers), operating at $5.712 \mathrm{GHz}$ (71 cells, $7 \mathrm{~mm}$ radius), with an average accelerating field of $\approx 35 \mathrm{MV} / \mathrm{m}$ [45].

Beam manipulation experimental studies have been performed to

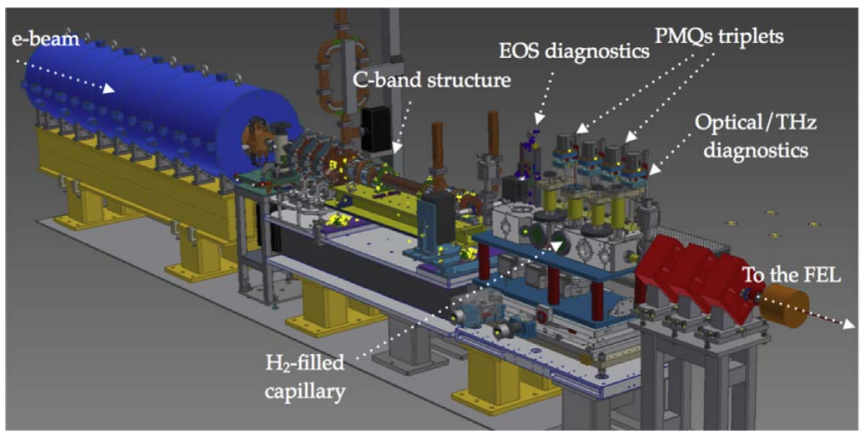

Fig. 4. Update layout of the photo-injector. The third S-band structure has been replaced by a $1.4 \mathrm{~m} \mathrm{C}$-band structure and the plasma interaction chamber, fully equipped with beam and plasma diagnostics and permanent quadrupole magnets. 


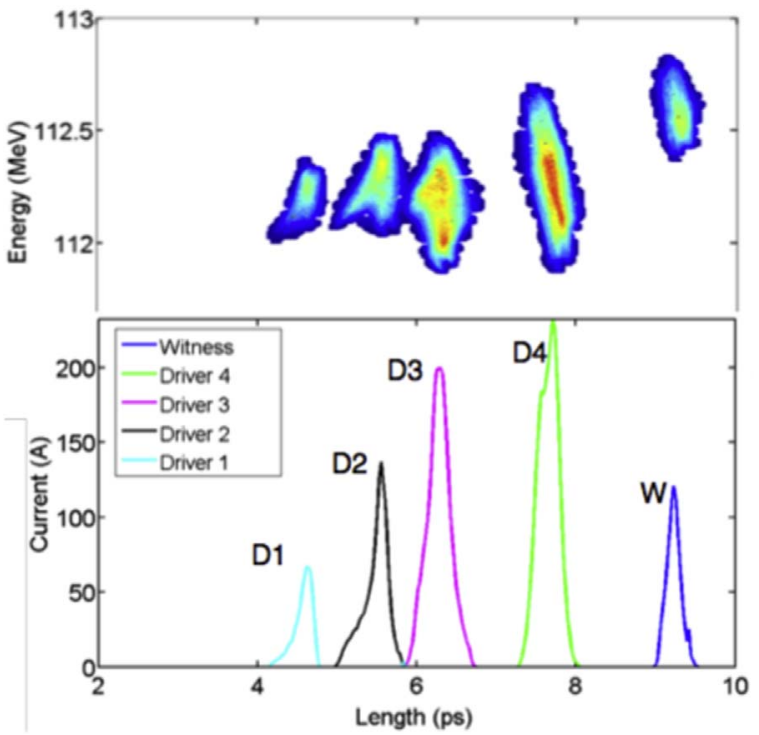

Fig. 5. Measured LPS of a comb-like beam consisting in a charge ramp four-driver bunches $(36,36,69,75 \mathrm{pC})$ and a witness $(24 \mathrm{pC})$. The witness bunch is at $3 \lambda_{p} / 2$ from the last driver (named as D4); the driver separation is $270-240-420 \mu \mathrm{m}\left(\lambda_{p}=330 \mu \mathrm{m}\right.$ with a plasma density $n_{0}=10^{16} \mathrm{~cm}^{-3}$ ).

Table 1

Single bunch measured parameters.

\begin{tabular}{llllll}
\hline Bunch name & $E(\mathrm{MeV})$ & $\Delta E / E(\%)$ & $\sigma_{t}(\mathrm{fs})$ & $Q(\mathrm{pC})$ & $\varepsilon_{n x}(\mathrm{~mm} \mathrm{mrad})$ \\
\hline $\mathrm{W}$ & 112.6 & 0.084 & 80 & 24 & $1(0.09)$ \\
D4 & 112.3 & 0.159 & 42 & 75 & $0.8(0.1)$ \\
D3 & 112.2 & 0.112 & 92 & 69 & $1.7(0.1)$ \\
D2 & 112.3 & 0.087 & 113 & 36 & $2.7(0.6)$ \\
D1 & 112.2 & 0.045 & 100 & 36 & $2.8(0.3)$ \\
\hline
\end{tabular}

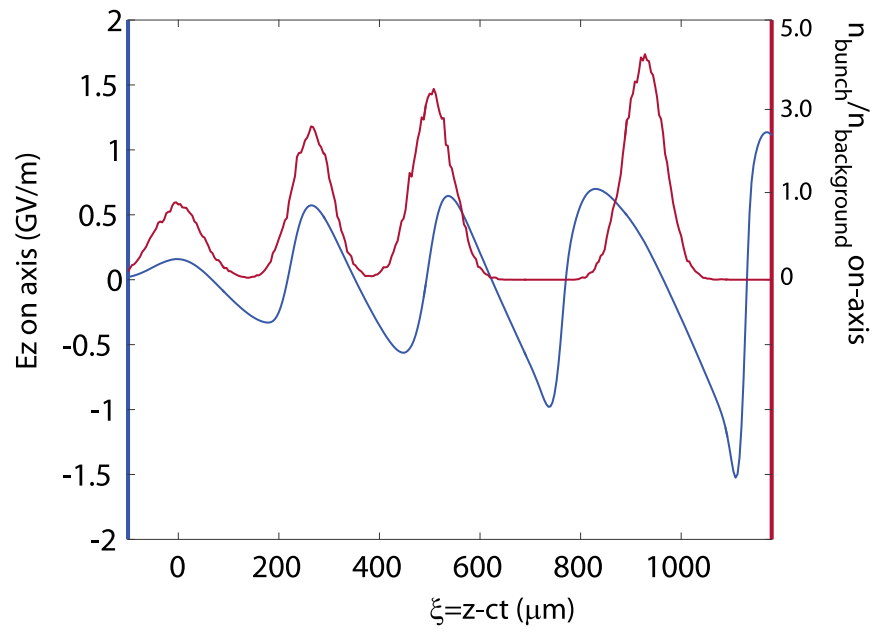

Fig. 6. Longitudinal electric field on axis (blue curve) and normalized bunch density profile (red curve), $\alpha=n_{b} / n_{0}$, for a ramp charge profile (ratio 1:3:5:7) in the weakly nonlinear regime: $\alpha \gg 1$ and $\widetilde{Q}=0.75$. The driver bunch spacing is non-uniform, following the experimental separation, i.e. $270-240-420 \mu \mathrm{m}\left(\lambda_{p}=330 \mu \mathrm{m}\right)$. The calculated transformer ratio is $R>3$. (For interpretation of the references to color in this figure caption, the reader is referred to the web version of this paper.)

optimize a multi-bunch ramped charge distribution in terms of temporal spacing, charge ratio, bunch length and transverse emittance. Fig. 5 shows the LPS of a four ramped drivers plus witness bunch train in the RF deep over-compression region, corresponding to $-100^{\circ}$ from the phase of maximum acceleration. The witness bunch $(24 \mathrm{pC})$ is at $3 \lambda_{p} / 2$ from the last driver (named as $D 4$ ); the driver separation is $\approx \lambda_{p}=330 \mu \mathrm{m}$ with a plasma density $n_{0}=10^{16} \mathrm{~cm}^{-3}$. Single bunch parameters are listed in Table 1; the normalized horizontal emittance has been measured by streaking the bunch train vertically with a RF deflecting cavity [46] (in parenthesis the statistical errors are reported). The error on the emittance measurement is not only statistical, but it takes into account also how big is the discrepancy between the model and the data. This is where the $10 \%$ error comes out. In addition, the fact that the fit does not follow precisely the data might derive from several reasons, e.g. RF breakdown during data acquisition, small signal-to-noise ratio, produced by the large magnification of the optics to distinguish beam dimensions of the order of few tens of $\mu \mathrm{m}$.

The fact that the inter-bunch spacing is not perfectly regular depends on the fixed distance of laser pulses at the cathode set by the crystal length. This drawback can be overcome by inserting delay lines for each laser pulse in order to adjust the final distance which is a combination of the compression phase too [26].

The effect of this non-uniformity on relevant parameters, i.e. transformer ratio and accelerating gradient, has been investigated and it is reported in Fig. 6. Fig. 6 shows the longitudinal electric field on axis (blue curve) and the normalized bunch density profile (red curve) for a ramped charge profile (ratio 1:3:5:7) in the weakly nonlinear regime: $\alpha \gg 1$ and $\widetilde{Q}=0.75$. The driver bunch spacing is nonuniform (contrary to what was shown in Fig. 2), following the experimental separation, i.e. $270-240-420 \mu \mathrm{m}$. Since $\lambda_{p}$ is $330 \mu \mathrm{m}$, it is clear that the first three bunches have all quite the same spacing, $\approx \lambda_{p}$, while the fourth is placed at $\approx 1.5 \lambda_{p}$, which is the position for optimized transformer ratio, but lower accelerating gradient (see Fig. 3). The calculated transformer ratio for the realistic case shown in Fig. 6 is slightly larger than 3; it means that, while the first three bunches, spaced $\approx \lambda_{p}$ apart, drive a $1.5 \mathrm{GV} / \mathrm{m}$ wake, the spacing of the last driver bunch, $\approx 1.5 \lambda_{p}$, contributes to preserve the transformer ratio. Hybrid spacing, or adjustable separation, might be a good compromise for the optimization of both accelerating gradient and energy transfer.

\section{Conclusions}

A future plasma-based user facility demands high brightness beams. The resonant amplification of plasma waves by a train of HBEBs injected into the preformed plasma is one of the schemes proposed to this scope. Manipulation of electron beam phase spaces is mandatory to enhance the energy transfer and preserve the beam quality. A preliminary characterization of the electron beam, manipulated to resonantly excite plasma wakes, has been performed at the SPARC_LAB test facility and results have been discussed.

\section{Acknowledgments}

This work has been partially supported by the EU Commission in the Seventh Framework Program, Grant agreement 312453 EuCARD2, by the Italian Ministry of Research in the framework of FIRB Fondo per gli Investimenti della Ricerca di Base, Project no. RBFR12NK5K and by the European Union's Horizon 2020 research and innovation programme under Grant agreement no. 653782. We wish to give a special thanks to S. Bini and V. Lollo for their contribution in the design and construction of the plasma chamber, and S. Pella for the plasma discharge circuit.

\section{References}

[1] J.B. Rosenzweig, et al., Experimental observation of plasma wake-field acceleration, Phys. Rev. Lett. 61 (1988) 98.

[2] S.P.D. Mangles, et al., Monoenergetic beams of relativistic electrons from intense laser-plasma interactions, Nature 431 (2004) 535-538.

[3] W.P. Leemans, et al., GeV electron beams from a centimetre-scale accelerator, Nat. Phys. 2 (2006) 696-699.

[4] I. Blumenfeld, et al., Energy doubling of $42 \mathrm{GeV}$ electrons in a metre-scale plasma wakefield accelerator, Nature 445 (2007) 741-744. 
[5] P. Muggli, et al., Witness bunch acceleration in a multi-bunch PWFA, in: Proceedings of 2011 Particle Accelerator Conference, New York, NY, USA, 2011.

[6] M. Litos, et al., High-efficiency acceleration of an electron beam in a plasma wakefield accelerator, Nature 515 (2014) 92-95.

[7] S. Steinke, et al., Multistage coupling of independent laser-plasma accelerators, Nature 530 (2016) 190-193.

[8] 〈http://www.eupraxia-project.eu〉.

[9] J.B. Rosenzweig, et al., Acceleration and focusing of electrons in two-dimensional nonlinear plasma wake fields, Phys. Rev. A 44 (10) (1991) R6189.

[10] J.B. Rosenzweig, et al., Plasma wakefields in the quasi-nonlinear regime: experiments at ATF, in: Advanced Accelerator Concepts: 15th Advanced Accelerator Concepts Workshop, vol. 1507, AIP Publishing: Austin, Texas, 2012, pp. 612-617.

[11] N. Barov, et al., Energy loss of a high-charge bunched electron beam in plasma: analysis, Phys. Rev. Spec. Top. - Accel. Beams 7 (2004) 061301.

[12] V.V. Tsakanov, On collinear wake field acceleration with high transformer ratio, Nucl. Instrum. Methods Phys. Res. Sect. A 432 (1999) 202-213.

[13] P. Chen, et al., Acceleration of electrons by the interaction of a bunched electron beam with a plasma, Phys. Rev. Lett. 54 (1985) 693;

P. Chen, et al., Acceleration of electrons by the interaction of a bunched electron beam with a plasma, Phys. Rev. Lett. 55 (1985) 1537.

[14] A. Marocchino, F. Massimo, Architect: First Release, 2016. http://dx.doi.org/10. 5281 /zenodo.49572.

[15] A. Marocchino, et al., Efficient modeling of plasma wakefield acceleration in quasinon-linear-regimes with the hybrid code Architect, Nucl. Instrum. Methods Phys. Res. Sect. A 829 (2016) 386-391.

[16] F. Massimo, S. Atzeni, A. Marocchino, J. Comput. Phys. 327 (2016) 841-850.

[17] K. Bane, P. Chen, P.B. Wilson, SLAC-PUB-3662, 1985

[18] R. Ruth, et al., A plasma wakefield accelerator, Part. Accel. 17 (1985) 171-189.

[19] W. Lu, et al., High transformer ratio PWFA for application on XFELs, in: Proceedings of 2009 Particle Accelerator Conference, Vancouver, BC, Canada, 2009.

[20] F. Massimo, et al., Transformer ratio studies for single bunch plasma wakefield acceleration, Nucl. Instrum. Methods Phys. Res. Sect. A 740 (2014) 242-245.

[21] F. Lemery, P. Piot, Tailored electron bunches with smooth current profiles for enhanced transformer ratios in beam-driven acceleration, Phys. Rev. Spec. Top. Accel. Beams 18 (2015) 081301.

[22] A. Mostacci, et al., Advanced beam manipulation techniques at SPARC, in: Proceedings of 2011 International Particle Accelerator Conference, San Sebastián, Spain, 2011.

[23] P. Muggli, et al., Generation of trains of electron microbunches with adjustable subpicosecond spacing, Phys. Rev. Lett. 101 (2008) 054801.

[24] Y. Shen, et al., Tunable few-cycle and multicycle coherent terahertz radiation from relativistic electrons, Phys. Rev. Lett. 107 (2011) 204801.

[25] M. Ferrario, et al., Laser comb with velocity bunching: preliminary results at SPARC, Nucl. Instrum. Methods Phys. Res. Sect. A 637 (2011) S43-S46.

[26] F. Villa, et al., Laser pulse shaping for high gradient accelerators, Nucl. Instrum.
Methods Phys, Res. Sect. A 829 (2016) 446-451.

[27] L. Serafini, M. Ferrario, Velocity bunching in photo-injectors, in: American Institute of Physics Conference Series, vol. 581, 2001, pp. 87-106.

[28] M. Ferrario, et al., Experimental demonstration of emittance compensation with velocity bunching, Phys. Rev. Lett. 104 (5) (2010) 054801.

[29] P. Piot, et al., Observation of coherently-enhanced tunable narrow-band terahertz transition radiation from a relativistic sub-picosecond electron bunch train, Appl. Phys. Lett. 98 (2011) 261501.

[30] M. Ferrario, et al., SPARC-LAB present and future, Nucl. Instrum. Methods Phys. Res. Sect. B 309 (2013) 183-188.

[31] M. Ferrario, et al., Direct measurement of the double emittance minimum in the beam dynamics of the sparc high-brightness photoinjector, Phys. Rev. Lett. 99 (2007) 234801.

[32] M.P. Anania, et al., Plasma production for electron acceleration by resonant plasma wave, Nucl. Instrum. Methods Phys. Res. Sect. A 829 (2016) 254-259.

[33] P. Tomassini, A.R. Rossi, Matching strategies for a plasma booster, Plasma Phys. Control. Fusion 58 (2016) 034001.

[34] C. Vaccarezza, et al., The SPARC LAB Thomson source, Nucl. Instrum. Methods Phys. Res. Sect. A 829 (2016) 237-242.

[35] F. Giorgianni, et al., Tailoring of highly intense THz radiation through high brightness electron beams longitudinal manipulation, Appl. Sci. 6 (2) (2016) 56.

[36] F. Giorgianni, et al., Strong nonlinear terahertz response induced by Dirac surface states in $\mathrm{Bi}_{2} \mathrm{Se}_{3}$ topological insulator, Nat. Commun. 7 (2016) 11421

[37] L. Giannessi, et al., Superradiant cascade in a seeded free-electron laser, Phys. Rev. Lett. 110 (2013) 044801.

[38] V. Petrillo, et al., Observation of time-domain modulation of free-electron-laser pulses by multipeaked electron-energy spectrum, Phys. Rev. Lett. 111 (2013) 114802.

[39] L. Giannessi, et al., Self-amplified spontaneous emission free-electron laser with an energy-chirped electron beam and undulator tapering, Phys. Rev. Lett. 106 (2011) 144801.

[40] A.R. Rossi, et al., The external-injection experiment at the SPARC_LAB facility, Nucl. Instrum. Methods Phys. Res. A 740 (2014) 60-66.

[41] R. Pompili, et al., Beam manipulation with velocity bunching for PWFA applications, Nucl. Instrum. Methods Phys. Res. Sect. A 829 (2016) 17-23.

[42] R. Pompili, et al., First single-shot and non-intercepting longitudinal bunch diagnostics for comb-like beam by means of electro-optic sampling, Nucl. Instrum. Methods Phys. Res. Sect. A 740 (2014) 216-221.

[43] E. Chiadroni, et al., The SPARC linear accelerator based terahertz source, Appl Phys. Lett. 102 (2013) 094101.

[44] E. Chiadroni, et al., Characterization of the THz radiation source at the Frascat linear accelerator, Rev. Sci. Instrum. 84 (2013) 022703.

[45] D. Alesini, et al., The C-Band accelerating structures for SPARC photoinjector energy upgrade, J. Instrum. 8 (2013) P05004.

[46] A. Cianchi, et al., Phys. Rev. Spec. Top. - Accel. Beams 18 (2015) 082804. 